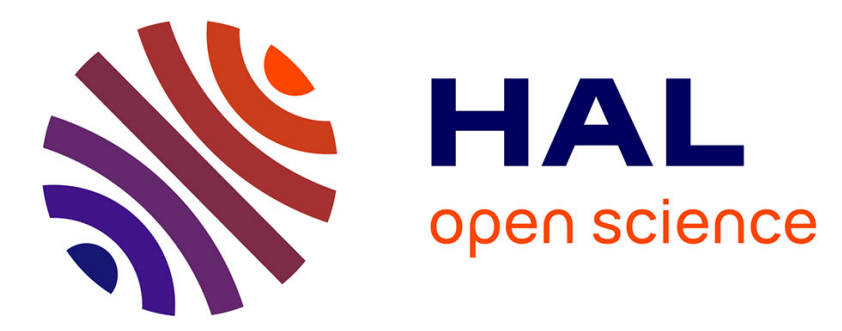

\title{
VibHand: On-Hand Vibrotactile Interface Enhancing Non-Visual Exploration of Digital Graphics
}

\author{
Kaixing Zhao, Marcos Serrano, Bernard Oriola, Christophe Jouffrais
}

\section{To cite this version:}

Kaixing Zhao, Marcos Serrano, Bernard Oriola, Christophe Jouffrais. VibHand: On-Hand Vibrotactile Interface Enhancing Non-Visual Exploration of Digital Graphics. Proceedings of the ACM on HumanComputer Interaction, In press. hal-02964264

\section{HAL Id: hal-02964264 https://hal.science/hal-02964264}

Submitted on 12 Oct 2020

HAL is a multi-disciplinary open access archive for the deposit and dissemination of scientific research documents, whether they are published or not. The documents may come from teaching and research institutions in France or abroad, or from public or private research centers.
L'archive ouverte pluridisciplinaire HAL, est destinée au dépôt et à la diffusion de documents scientifiques de niveau recherche, publiés ou non, émanant des établissements d'enseignement et de recherche français ou étrangers, des laboratoires publics ou privés. 


\title{
VibHand: On-Hand Vibrotactile Interface Enhancing Non- Visual Exploration of Digital Graphics
}

\author{
KAIXING ZHAO, IRIT, University of Toulouse, France \\ MARCOS SERRANO, IRIT, University of Toulouse, France \\ BERNARD ORIOLA, IRIT, CNRS, France \\ CHRISTOPHE JOUFFRAIS, IPAL, CNRS, Singapore
}

\begin{abstract}
Visual graphics are widely spread in digital media and are useful in many contexts of daily life. However, access to this type of graphical information remains a challenging task for people with visual impairments (VI). In this study, we designed and evaluated an on-hand vibrotactile interface that enables users with VI to explore digital graphics presented on tablets. We first conducted a set of exploratory tests with both people with VI and blindfolded (BF) people to investigate several design factors. We then conducted a comparative experiment to verify that on-hand vibrotactile cues (indicating direction and progression) can enhance the non-visual exploration of digital graphics. The results based on 12 participants with VI and 12 $\mathrm{BF}$ participants confirmed the usability of the technique and revealed that the visual status of the users does not impact graphics identification and comparison tasks.
\end{abstract}

CCS Concepts: • Human-centered computing $\rightarrow$ Accessibility; Empirical studies in accessibility; • Human-centered computing $\rightarrow$ Human computer interaction (HCI)

KEYWORDS: Interactive graphics; Vibrotactile interface; Accessibility; Visual impairment; Tactile display

\section{INTRODUCTION}

In 2018, the WHO estimated that there are 1.3 billion people with visual impairments (VI) and that 36 million of them are blind. It appears that access to graphical information (maps, schemas, mathematical graphs, drawings, etc.) is impaired due to difficulties in popularizing tactile adaptations of visual graphics (which process is based on methods mastered by professionals called tactile documents makers [35]). Therefore, apart from special education centers, tactile graphics are rare and expensive. In addition, another disadvantage of traditional tactile graphics is that they cannot be updated easily [13] and hence cannot meet the rapidly growing demand for graphical information. The lack of access to graphical representations can undermine the ability to conceptualize and learn from graphical data, and has an impact on education and mobility.

In recent years, many studies have focused on non-visual interactions for tactile (non-visual) exploration of digital graphics, which allow to take full advantage of the benefits of digital information (easily modifiable [15] and interactive [5]). Among these studies, various interaction modalities (for example haptic [34], audio [1] or multimodal [19]) were discussed to provide richer information. Recently, Tekli et al. [34] showed that it is possible to use the vibration of a tablet to explore a simple digital graphic. In their work, the tablet vibration is triggered, and stays on, as long as the exploring finger touches a segment of the graphic. Their study showed that VI users can understand basic shapes and simple geometrical objects.

Author's addresses: Kaixing Zhao, kaixing.zhao@irit.fr, IRIT, University Paul Sabatier, Toulouse, France; Marcos Serrano, marcos.serrano@irit.fr, IRIT, University Paul Sabatier, Toulouse, France; Bernard Oriola, bernard.oriola@irit.fr, IRIT, University Paul Sabatier, Toulouse, France; Christophe Jouffrais, christophe.jouffrais@irit.fr, IRIT, University Paul Sabatier, Toulouse, France. 
However, the absence of tactile cues under the fingertip when exploring digital graphics remains an issue (see Fig. 1-b). In contrast, when touching raised-line graphics, users can instantly perceive the direction of the line under the fingertip (see Fig. 1-a). This cue is important because it provides user with the direction of the next finger movement, which greatly facilitates the exploration process, especially for complex graphics.

In this study, we extended the idea of using the tablet vibration and explored if the use of guidance cues generated by on-hand vibrotactile interface can improve the exploration of complex digital graphics on touchscreens (see Fig. 1-c). Following an iterative and participatory design process, we investigated the use of a vibrotactile display on the back of the hand to convey two types of guidance cues: a pre-cue [12] for segment direction (see Fig. 1-d) and a confirmation cue concerning exploration progression (i.e. how far the finger is from the end of the segment, as illustrated on Fig. 1-e).

To this end, we first conducted a set of exploratory tests to investigate several design factors: the position of the interface on the hand, the duration of the vibrations, and the vibratory patterns used. These exploratory tests informed the design of the technique, called VibHand, which we then compared to the Tekli's technique [34] with 12 users with VI and 12 blindfolded users. The results showed that the proposed vibrotactile interface enables rapid and efficient exploration of complex graphics and improves the identification of the graphics. Interestingly, although there are differences according to the participants' visual status on exploration speed, we did not find any significant effect of user's visual status on digital graphic identification and comparison accuracy.

In summary, this article presents two main contributions: 1) A set of participatory studies to explore the properties of the back-of-the-hand vibrotactile interface assisting tactile exploration of digital graphics; and 2) An experiment involving 12 participants with VI and 12 blindfolded participants showing that the cues generated by the proposed vibrotactile interface improve (quicker and more efficient) the non-visual exploration of digital graphics.

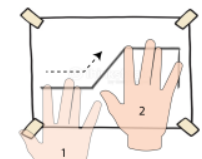

a)

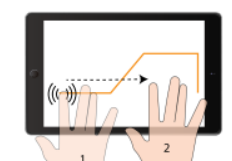

b)

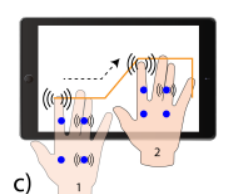

c)
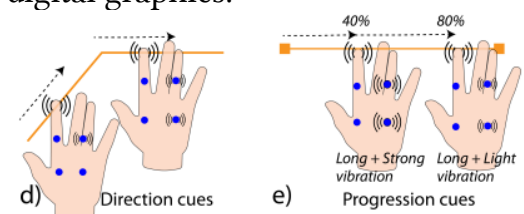

e)

Fig. 1. (a) Raised-line graphic exploration: tactile cues under the fingertip allow users to easily perceive line direction. (b) Digital graphic exploration: the tablet vibrates when the fingertip is over a digital line.

Exploration is difficult because of the absence of fingertip tactile cues. (c) VibHand: a back-of-the-hand vibrotactile feedback that provides users with direction (d) and progression (e) cues to improve the exploration of digital graphics on tablets.

\section{RELATED WORK}

\subsection{Tactile exploration of graphics}

Exploring graphics (raised-line or digital), is a common task for people with VI. Previous research has investigated how people with VI explore and understand two types of graphics: 1) "Line-independent" graphics and 2) "Line-based" graphics.

When exploring "line-independent" graphics, such as thematic maps, users generally do not need to carefully track the lines. Actually, they just need to perceive a border (even inaccurately) and they can then focus on the data contained in different areas of the graphic. They can rely on the kinesthetic perception of hand and finger movements in order to perceive the general layout of the graphic. Since several years, some researches $[6,13]$ have proposed to explore these graphics using audio-tactile methods. For instance, Bardot et al. [5] investigated 
the use of sparse vibratory and audio feedback to provide users with geospatial information, such as the demographics for a given region. The results showed that users are able to compare different areas and related data obtained by tactile exploration.

When exploring "line-based" graphics (e.g. understanding contours, paths or shapes), users can rely on kinesthetic cues but, as the integration of these cues is not precise enough, they must rely on the accurate tactile perception of lines too. Researches like [34] focused on this issue and proposed to use ongoing vibratory feedback to indicate the finger position. In this case, vibration is triggered when the user's finger touches the line and is stopped when the finger goes out of the line. The results show that this technique is usable for simple graphics but inappropriate for more complex graphics. The vibratory feedback does not compensate for the absence of tactile perception of the line orientation under the fingertip. It is then difficult to follow the lines and understand contours or shapes. In this work, we focused on the challenge of compensating tactile cues with vibrations applied on the hand.

\subsection{Interactive devices for digital graphic exploration}

According to the classification of Ducasse et al. [15], there are two types of interactive devices for digital graphic exploration: 1) hybrid devices that include both physical and digital components [16] and 2) devices that are fully digital [1, 5]. While hybrid devices are more convenient to use by people with VI because they can provide both tactile and kinesthetic cues, fully digital devices are easier to configure and update. In addition, fully digital graphics can be easily displayed on a commercial device like a tablet or mobile phone $[19,28]$. Finally, digital graphics can provide access to graphical data in many contexts including home, school, mobility, etc., without the need of a complex and expensive setup. In this work, we focused on how to enable complex digital graphics exploration on commercial tablets as a mean to provide ubiquitous access to graphical information.

\subsection{Interaction modalities for digital graphics exploration}

In previous work, the audio modality has been used to replace visual information and also to provide semantic description of visual graphics [25, 28]. Although these studies showed that audio can help people with VI to better understand the graphics, it is still an issue to describe the spatial layout of graphics based on audio descriptions only. In addition, as indicated in [36], the audio modality may not be appropriate in noisy or public environments and finding alternative solutions is essential for people with VI.

Several research works introduced other modalities to support richer and more flexible interactions, such as the abovementioned technique based on tablet vibration [34]. In addition, multimodal interactions are also frequently studied. For example, Goncu et al. [19] proposed a multimodal system to enable VI users to explore graphics based on audio and vibratory feedbacks. Similarly, Giudice et al. have proposed the use of simple vibrations coupled with audio to learn 2D graphics [18]. The use of multimodal interaction improved users' exploration to some extent but could also impair the exploration process due to the complexity of the feedback and increased cognitive load.

\subsection{Other vibrotactile interfaces}

Beyond access to graphics, there is an extensive literature in $\mathrm{HCI}$ on the use of vibrotactile feedback. Vibrotactile display [31] is a vibrant research topic in the domain of haptic interaction. It has been used in many contexts and has proved to be an efficient non-visual interaction method. For example, Chen et al. [11] systematically evaluated users' ability to recognize vibrotactile patterns generated by vibrotactile displays in a real-world environment. Another recent study focused on the perception of tactile cues applied on the wrist and fingers [17]. Both studies revealed that a vibrotactile display is an efficient way to transfer non-visual 
information, especially in a discrete and private way [10,22], which are important issues for people with VI.

In the domain of assistive technology for people with VI, several applications of vibrotactile displays have been proposed, such as visual context awareness [33], facial expressions recognition [21], virtual 3D shape identification [23], navigation [24] and localization of objects in the surroundings [12], etc. Among these studies, [20] evaluated wrist-based haptic feedback for non-visual target finding and path tracing on a 2D surface. In their study, they compared both the vibratory design (four versus eight vibrators) and vibration mode (single motor at a time versus two adjacent motors using interpolation, i.e. illusion). But none of these works have been applied to digital graphic exploration, where it could possibly compensate the lack of tactile direction cues under the fingertip.

\subsection{Summary and main objective}

The related work on "line-based" digital graphic exploration highlights the limitations of existing solutions based on ongoing vibrations, and the lack of studies on the use of more advanced vibrotactile interfaces for improving this non-visual exploration. In this work, our goal was to propose a novel vibrotactile interface to improve the exploration of line-based digital graphics based on the combination of the tablet vibration and a vibrotactile display attached to the back of the hand. We addressed the following research questions: 1) How to design a vibrotactile interface and tactile cues to improve non-visual tactile exploration of digital graphics? 2) Can people with VI use such a tactile interface to explore digital graphics without introducing additional cognitive load?

\section{PARTICIPATORY DESIGN}

For this work, we collaborated with a special education center for people with VI and adopted a participatory design approach [30] in which several people with VI participated to the design process. The whole design process was divided into two phases: 1) understanding users' needs and 2) designing the vibrotactile interface.

\subsection{Phase 1: Understanding users' needs}

We had many preliminary discussions with VI people and special education teachers to explore ideas of augmenting tactile exploration on tablets. To further discuss these ideas, we conducted a semi-structured interview with one special education teacher and three young adults with VI (trainees in the same institution, average age 29.1). During this session, we addressed the topics of tactile graphics, as well as audio and vibratory feedbacks to explore digital graphics. We summarize the main outcomes of the session below.

3.1.1 Audio tools. Using audio-based tools is very common with people with VI (e.g. screen readers for reading texts), and they are used to. The participants confirmed that audio description of graphics can improve comprehension [32], but also confirmed some issues already observed in the literature [36]. For instance, they explained that they must pay attention to surrounding sounds and people simultaneously, even in closed areas such as a classroom. In fact, they usually wear only one earphone. However, both sound sources can eventually be in conflict; then it becomes difficult to continue paying attention to audio descriptions and people at the same time. In this case, they have to make the choice to focus on one of the two sound sources. Therefore, if possible, they would prefer not to use audio-based enhancement while exploring graphics.

3.1.2 Choice of device and exploration cues. The participants of this session agreed that using a vibrotactile interface (e.g. vibrators on hands or arms) coupled with a regular tablet for presenting the digital graphics could be a valuable solution. A tablet is portable and can easily 
display graphics (easier than using tactile graphics obviously). A tablet is a good tradeoff between size and weight, especially for classroom or mobile situations. But the absence of tactile cues is an issue to understand graphics displayed on the screen. The vibratory feedback could fit the aim of guiding the hand towards a given direction.

3.1.3 Graphical information. The participants confirmed that in special education lessons, graphics (including maps, mathematical graphs, schemas, etc.) are simplified into coarse lines to be printed on a raised-line paper (see Fig. 2). As a first step into making digital graphics more accessible, it is possible to consider only linear segments (i.e. excluding curved lines) and eight segment directions (corresponding to the eight cardinal directions). Besides segment direction, providing progression (i.e. how much of a segment has been explored, as proposed in [8]) appeared as an interesting feature to facilitate the exploration of graphics with many segments.

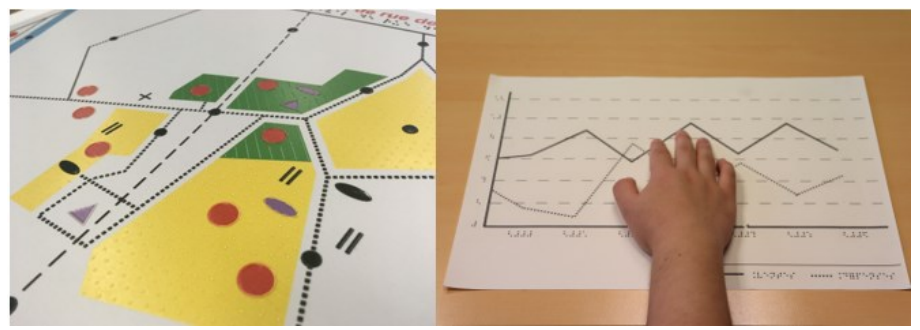

Fig. 2. Example of adapted raised-line graphics: geographic map (left) and mathematical graph (right).

\subsection{Phase 2: Designing the vibrotactile interface}

In this phase, we iteratively explored the different factors involved in the design of the vibrotactile interface. We conducted formative tests with 16 blindfolded (BF) participants (6 females and 10 males, $M=25.1, S D=2.7$ ) and two participants with VI (one 50 -year-old engineer with low-vision and one 53-year-old blind expert in HCI) to refine the technique. Here, our rationale for including blindfolded people in the formative tests was based on [26], which suggests that the ability to learn and mentally represent graphical material via vibrotactile feedback is similar between blindfolded users and users with VI. Relying on blindfolded subjects helps to reduce the constraints associated to the participation in experiments of people with VI (especially mobility issues and small number of available subjects [7]).

3.2.1 Position of the vibrotactile interface on the body. Vibratory feedback has been investigated on multiple body parts, including finger [17], waist [22], arm [11] and feet [24]. However, brainstorming sessions with the two VI subjects confirmed that the back of the hand was more convenient for tactile exploration for several reasons. The main one relies on the cognitive processes involved in hand guidance: if the tactile cues are applied on any other body parts, the user must mentally switch from the sensory reference frame (location of the tactile display) to the motor reference frame used for executing hand movements [12]. With the tactile display being fixed on the hand, there is an overlap between the sensory and motor reference frames. In addition, the back of the hand is always available and easy to access during tactile exploration of graphics. In contrast, the palm is in contact with the underlying surface, which makes it less suitable to accommodate the vibrators.

3.2.2 Numbers of vibrators. With the aim of representing eight cardinal directions, we may have used eight vibrators [3]. But due to limited space on the back of the hand and restriction of minimum inter-vibrator distance (for generating identifiable vibrations), we had to reduce the number of vibrators to four, and then use spatiotemporal vibrotactile patterns (SVP) [11] to increase the number of direction cues to eight (see Fig. 3 right). With this technique, we can use single vibrators to indicate the intermediate directions and two vibrators at the same time to indicate main directions. This design allowed us to render eight vibrotactile directions with four vibrators only, without exceeding the 2-bit limit for tactons mentioned in [17]. 
3.2.3 Vibration duration. As previously mentioned, direction cues are based on vibrations generated by one or two vibrators. One important aspect when using vibratory cues to assist graphic exploration is that the vibration duration should be as short as possible - although being unambiguously perceivable - in order to avoid slowing down the exploration process. We conducted a study to assess the vibration duration of each cue. We recruited 12 blindfolded participants and asked them to recognize the eight direction cues with different vibratory duration lengths $(50,150,200,250$ and $350 \mathrm{~ms})$. We compared two positions of the vibrators on the hand (A and $\mathrm{C}$ on Fig. 3). Each participant completed 160 trials corresponding to 2 vibrators positions * 8 directions * 5 durations * 2 repetitions. The Shapiro-Wilk test shows that the recognition rate follows a normal distribution $(\mathrm{p}=0.247)$. Then, we conducted an ANOVA which showed that there is no significant effect between the different vibration durations $(\mathrm{p}=$ .089). Therefore, we chose the mid value, i.e. $200 \mathrm{~ms}$, for our later studies.

3.2.4 Layout of the vibrators. The layout of the vibrators was another factor that we examined. Following the brainstorming sessions and formative tests (with three blindfolded participants and two participants with VI), we designed two layouts on the back of the hand based on Square and Cross shapes (Fig. 3 left). One important question during the design was about using the back of the hand only or including the fingers in the layout. The rationale for including the index finger comes from [17], which showed that recognition accuracy of vibrations applied to the index finger is good. We then proposed four configurations combining index finger and back-of-the-hand positions with Square and Cross shapes (see Fig. 3 Left). We conducted formative tests to select which of the four configurations is preferred and better conveys the eight vibrotactile directions. Overall, users preferred the Square layout because they found it was more obvious. They also preferred the vibrators on the fingers because they are located further away from each other, and it is easier to perceive and distinguish the vibrations on the fingers.

To further evaluate these layouts, we conducted a quantitative study. The task consisted in recognizing the direction of a $200 \mathrm{~ms}$ vibrotactile stimulus among the eight possible directions as quickly as possible. For this study, we recruited 16 blindfolded participants. Our study followed a within-subjects design with Configuration as the only factor (4 levels) as illustrated in Fig. 3 (A: Back-of-the-hand Square, B: Back-of-the-hand Cross, C: Finger-Hand Square and D: Finger-Hand Cross). The experiment included a familiarization session. Then the order of the conditions was counterbalanced, and the directional stimulus were randomly presented. Each participant completed 96 trials corresponding to 4 configurations * 8 directions * 3 repetitions. The average recognition rate with the four different configurations are: A $-82 \%, \mathrm{~B}-76 \%, \mathrm{C}-$ $93 \%$ and D $-82 \%$. (see Fig. 4). The Shapiro-Wilk test shows that the recognition rate follows a normal distribution $(\mathrm{p}=0.063)$. Hence, we conducted an ANOVA test which showed that there is a significant effect of the configuration $(\mathrm{p}<.001)$. The Tukey post-hoc showed that the recognition rate with configuration $\mathrm{C}$ was significantly superior than with configuration $\mathrm{A}(\mathrm{p}<$ $.01)$, B $(\mathrm{p}<.01)$ and $\mathrm{D}(\mathrm{p}<.01)$.

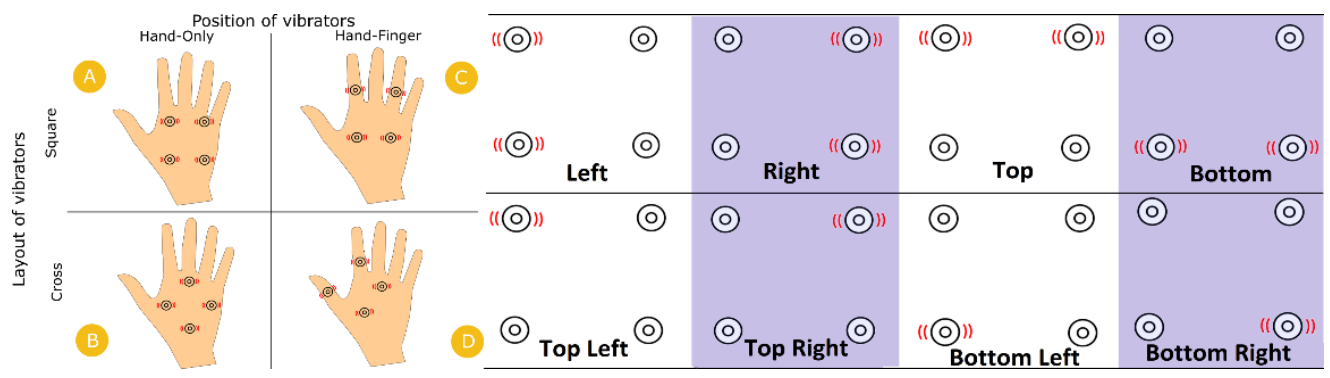

Fig. 3. Left: The four possible configurations according to the layout and position of vibrators on the back of the hand (A and B: square- and cross-shape respectively on the back of the hand; C and D: square- and 
cross-shape respectively on the back of the hand and fingers). Right: Illustration of the mapping between actuated vibrators and 8 direction cues for the Square layout.

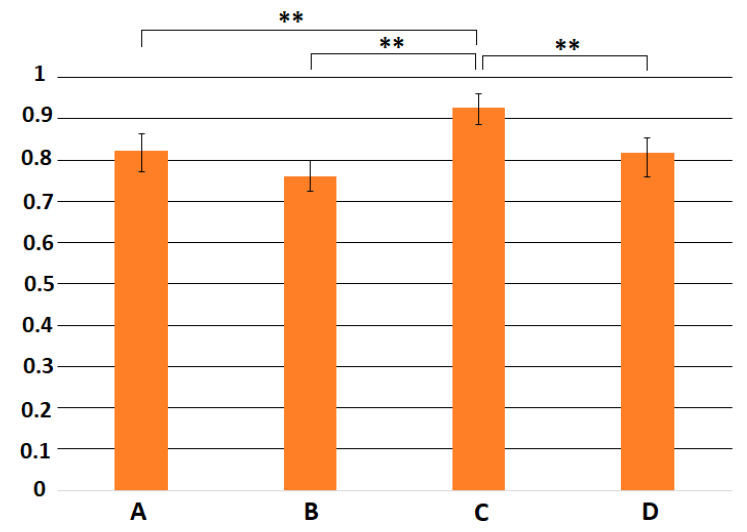

Fig. 4. Recognition rate with different configurations $(95 \% \mathrm{Cl})$.

3.2.5 Vibrotactile progression cues. To represent the progression information (i.e. how far is the end of the segment), it was important to design a technique compatible with the direction cues to avoid any confusion. First of all, we defined the duration of the progression cues to 500 ms (i.e. more than twice longer than the direction cues). This choice was inspired by [8] which applied vibrations of $600 \mathrm{~ms}$ on the wrist. Since the hand is more sensible than the wrist, we decided to reduce this value to $500 \mathrm{~ms}$, as our goal was to have the shortest possible vibrations so as not to slow down the exploration task.

Next, we considered how to design the progression cues and we identified two cases according to the number of cues provided for a segment. In the first case, we used four successive vibrations located respectively at $20 \%, 40 \%, 60 \%$ and $80 \%$ of the segment and with increasing or decreasing intensity. In the second case, the progression cue was triggered only twice, at $40 \%$ and $80 \%$ of the segment with also increasing or decreasing intensity (see Fig. 1-e). We conducted a test which consisted on exploring a single segment on different directions with progression cues. We recruited four blindfolded participants who had taken part in the study on vibration duration. Each participant completed 96 trials corresponding to 2 vibration patterns (two vs. four stimuli) * 2 vibration modes (increasing vs. decreasing) * 8 directions * 3 repetitions. The results based on a 5-level Likert scale showed that all four participants preferred using two progression cues (instead of four) and three out of four participants preferred progression cues with decreasing intensity (instead of increasing).

3.2.6 Cues triggering. The direction cue is triggered as soon as the fingertip touches a line on the graphic. If the finger arrives in the middle of a segment, the direction cue to the furthest end is chosen. As explained earlier, the progression cue appears at a fixed percentage of the segment ( $40 \%$ and $80 \%$ of the segment).

3.2.7 Graphic line width. We initially decided to use the same line width as previous work on digital graphics (8.9 $\mathrm{mm}$ in [34]). However, after preliminary tests, we found that such width: 1) is far too large to represent complex graphics on a tablet, 2) is larger than raised-lines on tactile documents (usually they are about $2 \mathrm{~mm}$ width), and then does not correspond to the experience of exploring raised-line graphics. Hence, we reduced the line width according to the results of [26]. With a $4 \mathrm{~mm}$ line width, participants can explore digital segments on touchscreens without perceptual issues. Obviously a $4 \mathrm{~mm}$ line width is more compatible with complex (meaningful) graphics displayed on 10 inches' commercial tablets. 


\section{EXPERIMENT: EXPLORING AND IDENTIFYING GEOMETRICAL AND NON- FIGURATIVE GRAPHICS}

The aim of this behavioral experiment was to verify the general hypothesis that the two cues rendered by the vibrotactile interface can enhance digital graphics exploration without introducing additional perceptual or cognitive issues.

\subsection{Participants}

In line with [26], we included blindfolded sighted subjects in our experimental design to assess for any differences on the exploration performance between people with VI and blindfolded sighted people due to cognitive skills. Blindfolded sighted participants can be considered as novices in tactile exploration. We recruited 24 participants that were divided in two groups according to their visual status: BF group (12 blindfolded participants, 2 females, 10 males) aged between 21 and $28(\mathrm{M}=25.1, \mathrm{SD}=1.9)$; and VI group (12 people with VI, 6 females, 6 males) aged between 25 and $60(\mathrm{M}=45.9, \mathrm{SD}=12.2)$. None of the VI and BF participants (described in Table 1) presented any additional sensory impairments nor took part in the previous studies.

Table 1. Description of the VI and BF participants

\begin{tabular}{|c|c|c|c|c|c|c|c|}
\hline ID & Gender & Age & Description & ID & Gender & Age & Description \\
\hline VI 01 & M & 27 & $\begin{array}{c}\text { Engineer, Blind from } \\
\text { age } 12\end{array}$ & $\mathrm{BF} 01$ & M & 21 & $\begin{array}{c}\text { Undergraduate } \\
\text { student }\end{array}$ \\
\hline VI 02 & $M$ & 54 & $\begin{array}{l}\text { Special education } \\
\text { teacher, blind form } \\
\text { age } 12\end{array}$ & $\mathrm{BF} 02$ & M & 28 & Ph.D. student \\
\hline VI 03 & $\mathrm{~F}$ & 59 & $\begin{array}{l}\text { Special education } \\
\text { teacher, blind from } \\
\text { birth }\end{array}$ & BF 03 & M & 23 & $\begin{array}{c}\text { Undergraduate } \\
\text { student }\end{array}$ \\
\hline VI 04 & $\bar{M}$ & 28 & $\begin{array}{l}\text { Unemployed, blind } \\
\text { from birth }\end{array}$ & $\mathrm{BF} 04$ & $\mathrm{M}$ & 26 & Master student \\
\hline VI 05 & $\mathrm{~F}$ & 48 & $\begin{array}{l}\text { Special education } \\
\text { teacher, low vision } \\
\text { from age } 11\end{array}$ & $\mathrm{BF} 05$ & $M$ & 25 & Master student \\
\hline VI 06 & $\mathrm{~F}$ & 60 & $\begin{array}{l}\text { Teacher, low vision } \\
\text { from birth }\end{array}$ & BF 06 & M & 23 & Master student \\
\hline VI 07 & $\mathrm{~F}$ & 50 & $\begin{array}{l}\text { Special education } \\
\text { teacher, very low } \\
\text { vison from birth } \\
\end{array}$ & $\mathrm{BF} 07$ & $M$ & 26 & Ph.D. student \\
\hline VI 08 & $\bar{M}$ & 45 & $\begin{array}{l}\text { Unemployed, blind } \\
\text { from birth }\end{array}$ & BF 08 & $\mathrm{~F}$ & 25 & Master student \\
\hline VI 09 & $\mathrm{M}$ & 44 & $\begin{array}{l}\text { Unemployed, very } \\
\text { low vision from birth }\end{array}$ & $\mathrm{BF} 09$ & $M$ & 26 & Engineer \\
\hline VI 10 & $\mathrm{M}$ & 59 & $\begin{array}{c}\text { Engineer, blind from } \\
\text { age } 6\end{array}$ & $\mathrm{BF} 10$ & $M$ & 27 & Master student \\
\hline VI 11 & $\mathrm{~F}$ & 52 & $\begin{array}{l}\text { Teacher, very low } \\
\text { vision from birth }\end{array}$ & BF 11 & $\mathrm{~F}$ & 26 & Ph.D. student \\
\hline VI 12 & $\mathrm{~F}$ & 25 & $\begin{array}{l}\text { Student, very low } \\
\text { vision from birth }\end{array}$ & BF 12 & M & 25 & Engineer \\
\hline
\end{tabular}

\subsection{Task and instructions}

We used a Delayed-Matching-to-Sample (DMTS) task [14], which consists in comparing pairs of stimuli and is widely used in working memory studies. In our case we asked participants to 
explore and compare pairs of digital graphics. More precisely, for each trial, the participant was first presented with a sample stimulus (sample graphics) and then an alternative stimulus (alternative graphic) after a short delay. The exploration of each pair included three steps:

1) Encoding phase: participants were asked to completely explore the sample graphic as quickly and accurately as possible. After the exploration, and for the geometrical graphics only, they had to verbally identify the shape, e.g. "square" (identification task).

2) Retention delay: a short delay between the two stimuli. Here, we chose a 5 s retention delay to elicit the use of memory strategies [9].

3) Test phase: participants were asked to explore the alternative graphic as quickly and accurately as possible. They were free to stop the exploration as soon as they can decide that the pair is identical or different (comparison task).

\subsection{Digital graphics}

We selected two different types of digital graphics: Simple Geometrical Graphics (shown in Fig. 5, sets A and B) and Non-Figurative Graphics (shown in Fig. 5, sets C and D).

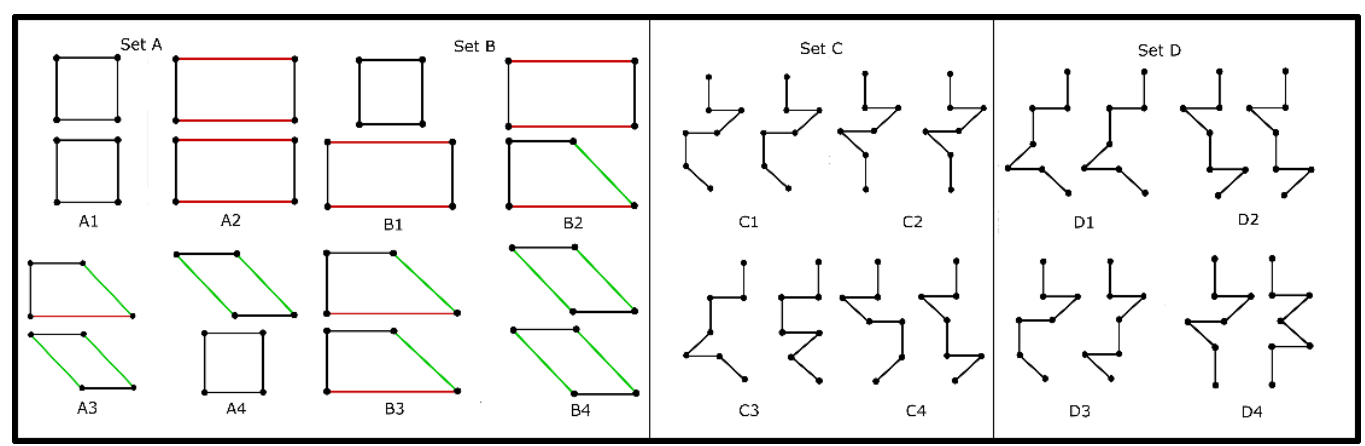

Fig. 5. Sets A and B: Geometrical graphic pairs, Sets C and D: Non-Figurative graphic pairs.

Simple Geometrical Graphics (G-Graphics): these digital graphics are common geometrical shapes that were selected by meeting three criteria: 1) Same complexity: all the selected graphics have the same numbers of segments. 2) Similar shape: in order to design a DMTS task that was not too simple, the selected graphic pairs have at least two identical segments (but subjects were not aware of that). 3) Commonly used: all the graphics are known by users. Finally, the G-Graphics group included four common quadrilaterals: Square, Rectangle, Right Angle Trapezoid and Parallelogram.

Non-Figurative Graphics (NF-Graphics): these digital graphics were 2-D patterns made from a combination of 6 segments ( 2 horizontal, 2 vertical, 1 slash and 1 backslash). The use of such patterns was inspired by [4], which mentioned that, contrary to geometric shapes, the tactile exploration of 2D non-figurative patterns is not dependent on existing mental representation of the shape. Like geometrical graphics, we designed "identical" and "similar" pairs. Here, similar pairs mean that the two graphics started with three same segments and always differed on the fourth or fifth segment (but subjects were not aware of that).

\subsection{Interaction techniques}

We compared the VibHand technique, described in the previous section, with a touchscreen vibration technique for tablets proposed by Tekli et al. [34] as the control condition (for more details see the related work section). While our technique provides more cues than the control condition (which is based on touchscreen vibration only), we aimed to check whether introducing additional vibrations may increase cognitive load and then decrease the performance. Concerning the inclusion of other modalities, our initial analysis showed that the 
audio channel is overloaded in Assistive Technologies and that audio guidance can interfere with surrounding sounds and speech. Therefore, we discarded to include any audio-based solution in our study.

\subsection{Experimental design}

We used a within-subjects design with three factors: the interaction technique (VibHand vs. Control), the type of graphic (G-Graphics vs. NF-Graphics) and participants' visual status (BF vs. VI).

The study included two familiarization phases followed by two test phases. During the first familiarization phase, participants were presented with vibrotactile cues along eight directions: Top, Bottom, Left, Right, Top Left, Top Right, Bottom Left and Bottom Right. They were free to practice several times in each direction with both techniques. During the second familiarization phase, participants were asked to explore carefully four digital segments: Horizontal, Vertical, Slash and Backslash using both interaction techniques. They were free to practice several times until they felt confident with both interaction techniques.

Then the session was divided into two blocks corresponding to the two types of digital graphics (first block with the G-Graphics and second block with NF-Graphics), i.e. with increasing difficulty. In each block, the participants explored the graphics with the control technique first and then VibHand, or vice versa. We counterbalanced the order of interaction techniques across participants. It is important to note that although we included the type of graphics as a factor in our study, our analysis does not intend to look for differences across both types of graphics (because they have different complexity and length), but rather look for interactions with the other factors (interaction technique and participant's visual status).

\subsection{Experimental setup}

During the experiment, participants were asked to comfortably sit in front of a Samsung Galaxy Tab S4 (10.5 inch, $1600 \times 2560$ px, 287 ppi density). The vibrotactile cues (VibHand) were generated by four vibrators (model RB-See-403, SeeedStudio) attached on the back of the hand (see Fig. 6). The vibration (intensity and duration) was controlled by the Pulse Width Modulation (PWM) of the Arduino UNO, which was wire connected to a nearby laptop (we ensured that the wires did not interfere with hand movements).

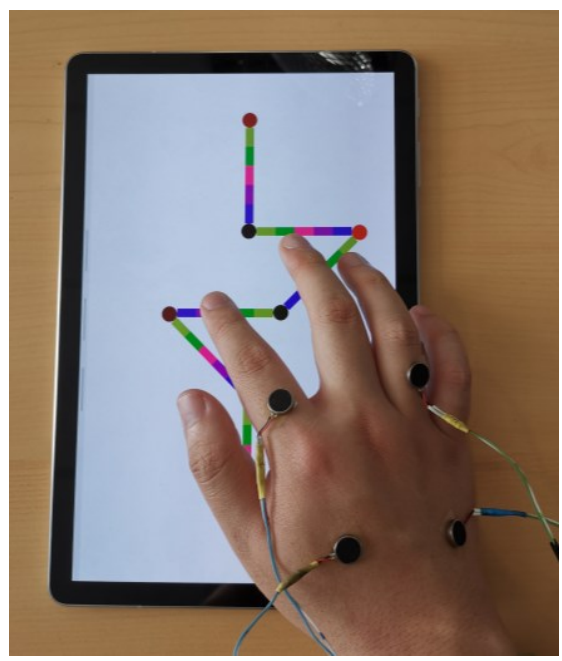


Fig. 6. Experimental setup with a NF-Graphic displayed on the tablet and the four vibrators attached to the back of the hand.

\subsection{Collected data and dependent variables}

We recorded the finger path during the exploration of each graphic. We computed different dependent variables: the exploration speed in $\mathrm{cm} / \mathrm{s}$ for each sample graphic; the unrelated finger path as the difference in $\mathrm{cm}$ between users' real finger path and the length of the graphic; the time needed to compare the pair (response time); the graphic identification accuracy (for the sample graphic on the geometrical set); and comparison accuracy (for each pair). We also collected the subjective rating (on a 5-point Likert scale) about the following questions: a) Is VibHand easy to use? b) Does VibHand make the exploration easier? c) Are progression cues useful? and d) Do you prefer VibHand or Control?

In total, we collected 24 (12 blindfolded participants +12 participants with VI) * 4 (2 sets of geometrical graphics +2 sets of non-figurative graphics) * 4 (4 pairs of graphics in each set) $=$ 384 trials $(1$ trial $=1$ pair). Since the alternative graphic was not systematically completely explored, these trials represent between 1536 segments (if only the sample graphics were explored) and 3072 segments (if all the alternative graphics were fully explored).

\subsection{Results}

In total, participants explored 2716 segments (i.e. $88.4 \%$ of the total set of segments).

4.8.1 Identification of G-Graphics. According to the Shapiro-Wilk test, the average identification rate does not follow a normal distribution $(\mathrm{p}<.001)$. Since the raw data of this measure is dichotomous, we adopted a Chi-Squared test. The results show a significant effect of the interaction technique $\left(X^{2}=20.841, \mathrm{p}<.001\right)$ but no effect of the visual status $\left(X^{2}=.2573, \mathrm{p}=\right.$ .612). The identification accuracy is better with VibHand than with Control ( $90.6 \%$ vs. $61.4 \%$, see Fig. 7). Identification errors generally corresponded to participants mixing square with rectangle, and trapezoid with parallelogram.

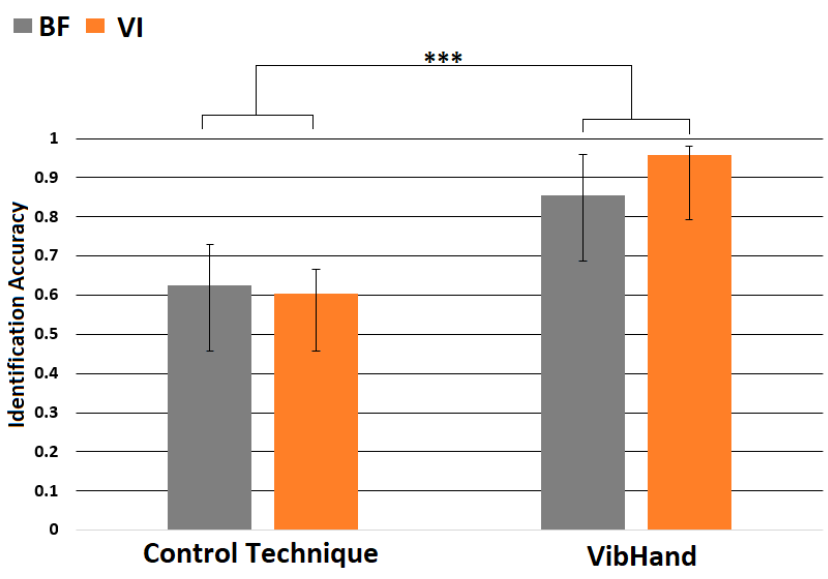

Fig. 7. Average identification accuracy for G-Graphics $(95 \% \mathrm{CI})$.

4.8.2 Pair comparison. The pair comparison accuracy does not follow a normal distribution according to the Shapiro-Wilk test $(\mathrm{p}<.001)$. Similarly, since the raw data of this measure is dichotomous, we also adopted a Chi-Squared test. The results show a significant effect of the interaction technique $\left(X^{2}=18.665, \mathrm{p}<.001\right)$ but no effect of the visual status $\left(X^{2}=.921, \mathrm{p}=.337\right)$ nor the graphic type $\left(X^{2}=1.44, \mathrm{p}=.23\right)$. Overall, the comparison accuracy is better with VibHand than with Control (85.9\% vs. 66.6\%, Fig. 8). 


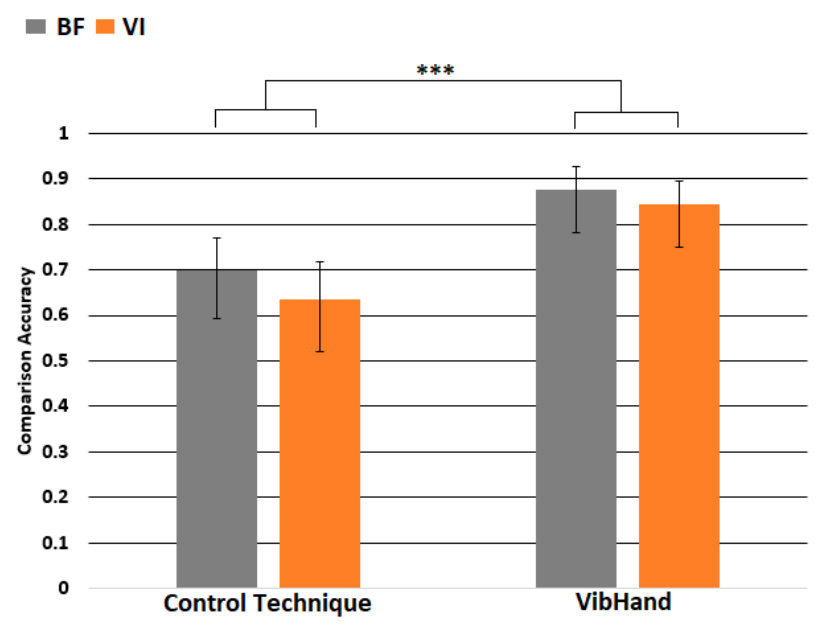

Fig. 8. Average comparison accuracy $(95 \% \mathrm{Cl})$.

4.8.3 Response Time (to judge same or different). This measure is based on the exploration time of alternative graphics (i.e. the second graphic of each pair), which reflects on the time needed to explore the alternative graphic and judge if it is identical or different. The distribution was not normal according to the Shapiro-Wilk test $(\mathrm{p}<.001)$. However, after a Box-Cox transformation [29], the response times finally follow a normal distribution $(\mathrm{p}=.84)$. Then the results of the ANOVA showed that there is a main effect of the interaction technique $(\mathrm{F}(1,11)=$ $16.6, p=5 e-5)$ and of the graphic type $(F(1,11)=6.9, p=.00883)$, but no effect of the visual status $(\mathrm{F}(1,11)=1.49, \mathrm{p}=.22)$. There was also an interaction between the type of graphic and the visual status $(\mathrm{F}(1,11)=4.05, \mathrm{p}=.045)$. The average response time is shorter with VibHand than with Control (42.0 s vs. 53.4 s, see Fig. 9), which shows that it is easier to make the comparison with VibHand. It is also shorter for G-Graphics than for NF-Graphics (45.2 s vs. 50.2 s).

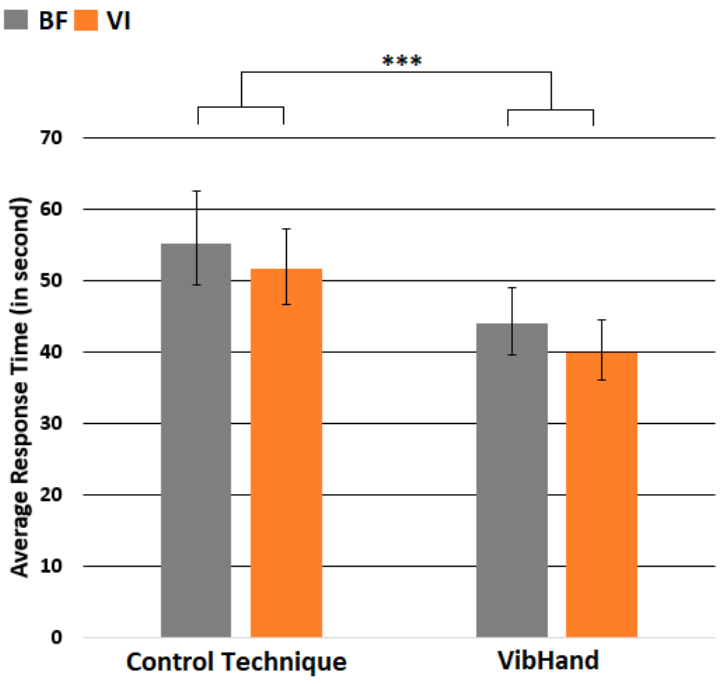

Fig. 9. Average response time $(95 \% \mathrm{Cl})$. 
4.8.4 Unrelated finger path for the sample graphic. The Shapiro-Wilk test shows that the average unrelated finger path does not follow a normal distribution $(p<.001)$. Hence, we transformed the data to normal using Box-Cox $(\mathrm{p}=.096)$. The ANOVA shows a significant effect of the interaction technique $(\mathrm{F}(1,11)=248.94, \mathrm{p}=2 \mathrm{e}-16)$ and visual status $(\mathrm{F}(1,11)=$ $19.56, \mathrm{p}=1 \mathrm{e}-7)$ on the unrelated finger path, but no effect of the type of graphic $(\mathrm{F}(1,11)=0.25$, $\mathrm{p}=0.62)$. We also found an interaction effect between two pairs of factors: interaction technique * type of graphic $(\mathrm{F}(2,21)=5.29, \mathrm{p}=0.022)$ and visual status * type of graphic $(\mathrm{F}(1$, $11)=3.93, \mathrm{p}=0.0483)$. In fact, VibHand decreases the average unrelated paths $(45.0 \mathrm{~cm}$ vs. 132.8 $\mathrm{cm})$ as illustrated in Fig. 10. The average unrelated finger path was smaller for VI participants than for BF participants $(70.9 \mathrm{~cm}$ vs. $106.9 \mathrm{~cm})$. The Tukey post-hoc test shows significant effects on both Simple Geometrical Graphics and Non-Figurative Graphics (for both BF and VI groups, the $\mathrm{p}<.001)$.

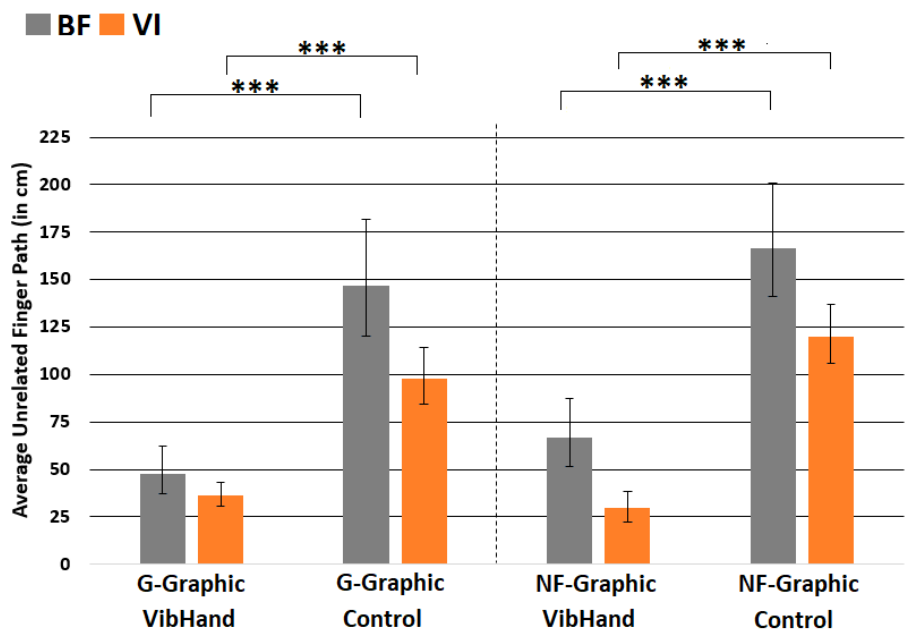

Fig. 10. Average unrelated finger path $(95 \% \mathrm{Cl})$.

The interaction effects show that the differences are always more pronounced on nonfigurative graphics: VibHand performed better on NF-Graphics than on G-Graphics compared to Control. This effect shows that it is easier to explore NF-Graphics with VibHand and it could just be related to the fact that NF-Graphics are always explored in the second block (learning effect).

4.8.5 Exploration speed. Since the two types of digital graphics do not have the same complexity (the number of segments for geometrical and non-figurative graphics are 4 vs. 6 respectively), we conducted the statistical analysis on exploration speed (and not exploration time).

The Shapiro-Wilk test shows that the exploration speed does not follow a normal distribution $(\mathrm{p}<.001)$. Hence we transformed the data using Box-Cox $(\mathrm{p}=.086)$. The ANOVA shows that there is a significant effect of visual status $(\mathrm{F}(1,11)=6.34, \mathrm{p}=0.0122)$, interaction technique $(\mathrm{F}(1,11)=75.47, \mathrm{p}=2 \mathrm{e}-16)$ and graphic type $(\mathrm{F}(1,11)=4.8, \mathrm{p}=0.029)$ on exploration speed, but no interactions between factors. For both BF and VI participants, the exploration speed is faster with VibHand than with the control technique $(0.61 \mathrm{~cm} / \mathrm{s}$ vs. $0.42 \mathrm{~cm} / \mathrm{s}$, see Fig. 11-b) and participants with VI explore graphics on average faster than BF participants $(0.54 \mathrm{~cm} / \mathrm{s}$ vs. $0.48 \mathrm{~cm} / \mathrm{s}$, see Fig. 11-a). In addition, the average exploration speed of G-Graphics and NFGraphics are $0.50 \mathrm{~cm} / \mathrm{s}$ and $0.53 \mathrm{~cm} / \mathrm{s}$ respectively (see Fig. 11-c). 


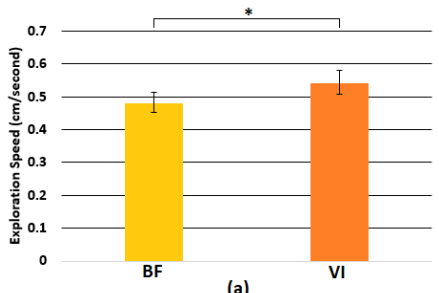

(a)

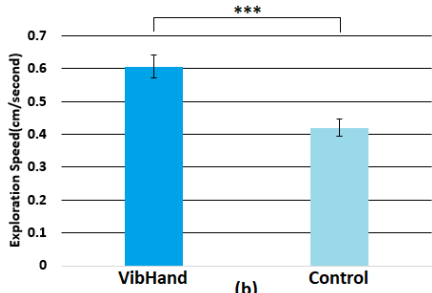

(b)

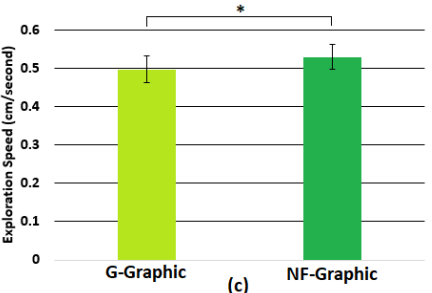

(c)

Fig. 11. Average exploration speed $(95 \% \mathrm{Cl})$ by a) visual status, b) interaction technique and c) graphic type.

4.8.6 Exploration movements. In addition to the previously described measures, we analyzed the overall finger path observed with the two techniques. We observed numerous inefficient exploration movements with the control technique. For example, when reaching the end of a segment with this technique, participants came up with two strategies to find the next path: doing a circular movement with the finger until finding the next segment (Fig. 12A, participant VI 02); or initiating a finger movement in many different directions, drawing a "star like" path (Fig. 12B, participant VI 06). Obviously, both "circles" and "stars" waste time and increase the unrelated finger paths. In addition, they are probably confusing too, which can also explain worse identification and comparison scores.

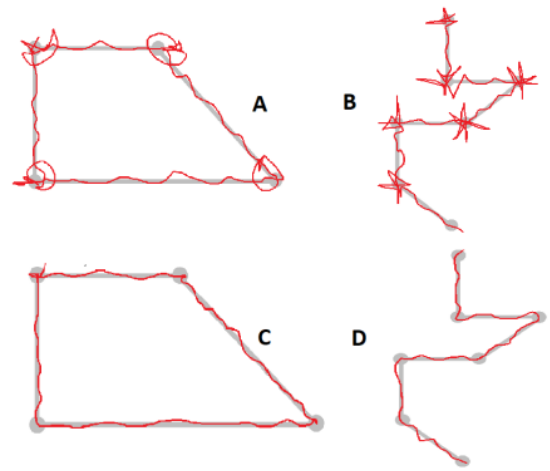

Fig. 12. A and C: VI 02 finger paths when exploring a geometrical graphic with control technique and VibHand respectively; B and D: VI 06 finger paths when exploring a non-figurative graphic with control technique and VibHand respectively.

The comparison of the finger paths with VibHand clearly shows that the reduction in unrelated finger path comes from: a) more linear paths when exploring segments, and b) more efficient transitions at the end of segments.

4.8.7 Qualitative feedback. Eleven out of $12 \mathrm{VI}$ participants strongly agree or agree that the vibrotactile directional cues are easy to use and make the exploration easier. Nine strongly agree or agree that the progression cues are useful, and eleven prefer exploring with VibHand. Fig. 13 shows the responses of participants with VI. The only participant with VI who disagreed (VI 04) explained that "Personally I don't like the vibration. Even for my mobile phone, I don't activate the vibration mode, I'm kind of special!".

Verbatim recorded during the experiment were useful to understand the feelings of the participants. For example, while exploring with VibHand, VI 09 said "I understand! I know how to translate the vibration now and it's really cool"! In contrast, exploring the same graphic with the control technique, he said "It's really hard, how do you follow the lines? I can't do this." 
Similar reactions occurred also to many other participants (both blindfolded participants and participants with VI).

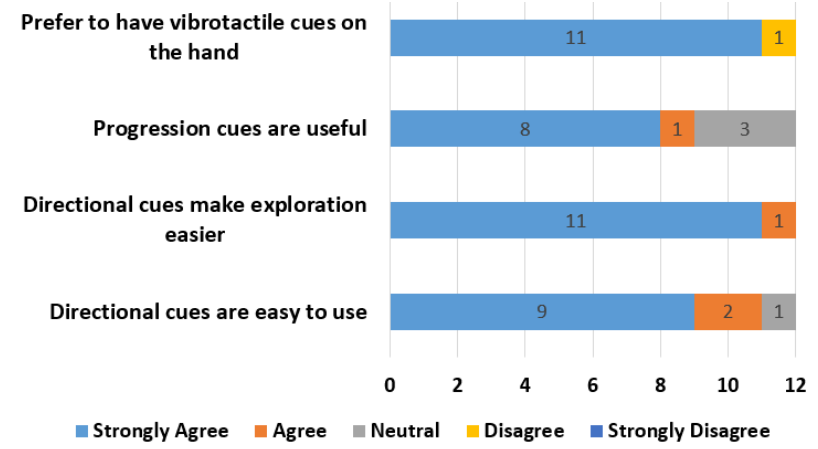

Fig. 13. Qualitative feedback of VI participants for the VibHand technique.

\subsection{Discussion}

4.9.1 Vibrotactile cues improve digital graphic exploration. The results confirm our hypothesis that VibHand improves non-visual digital graphic exploration (for both people with VI and blindfolded sighted people). It improves both the recognition accuracy of known graphics and the comparison between two graphics. In addition, it reduces the unrelated finger path, increases the exploration speed and also decreases the time needed to compare two graphics. The comparison of the finger paths with VibHand shows that the reduction in unrelated finger path comes from: a) more linear paths when exploring segments, and b) more efficient transitions at the end of segments. Finally, although this result may still need to be confirmed with a standardized test of cognitive load (for example, NASA-TLX or SAGAT, etc.), the qualitative feedback and verbatim indicate that the technique does not introduce additional perceptual or cognitive load during exploration.

4.9.2 Impact of user's visual status. By comparing BF and VI participants, we did not observe any difference on the identification accuracy of known graphics, nor on the comparison accuracy. Both blindfolded sighted and subjects with VI were able to do the task with good accuracy, which is in line with [26]. However, the results show that VI participants take less time and have shorter unrelated finger paths than BF participants. The most probable explanation is that the difference is not perceptual but cognitive. The results show that both VI and $\mathrm{BF}$ participants were able to rely on the direction and progression cues to explore digital graphics but VI participants were more efficient. In fact, VI people are used to explore raisedline graphics, as well as digital contents on smartphones or tablets, and can be considered as experts in tactile exploration. Our interpretation is that they rely on better cognitive skills than $\mathrm{BF}$ participants: they can better integrate perceptual cues in time and space in order to form a mental representation of the displayed graphics.

This result also means that there is no advantage for blindfolded sighted people, in these two specific tasks, although it has been shown that vision helps to get better mental representation of shapes. In fact, it could be that these two effects cancel each other. More research work should address this question.

Finally, we recruited participants with VI and blindfolded sighted participants having different educational background and age. It appears that the blindfolded sighted participants are, on average, younger and with a higher educational background. Although we believe that these differences in education and age should be in favor of the blindfolded sighted participants in the tasks that we proposed, the question should be addressed in a future work.

4.9.3 Application scenarios. In our work, we evaluated relatively simple graphics (but more complex than those in [34]). However, VibHand can easily be applied to different real scenarios. 
We acknowledge that all the scenarios including VibHand require a simplification of the graphics first (segments along the eight cardinal directions only). However, it is important to note that this is already the case when creating raised-lines graphics for people with VI [35]. Here, we present and discuss two possible applications scenarios (among many others):

Digital map exploration for orientation and mobility lessons. During the collaboration with the special education center, we found that neighborhood maps are used almost daily in classes of Orientation \& Mobility. A frequent request of students with VI is to have access to these maps in different situations (at home, in a public building, in an unknown place, etc.). Using a regular tablet with VibHand, users with VI could access to digital graphics everywhere. Fig. 14 illustrates a simplified digital neighborhood map, where each line represents a road. VibHand can be used to explore the map and provide users with better knowledge of the paths between two points of interest (e.g. between the subway exit and the school). In fact, although people with VI already use raised-line maps in such situations, during O\&M classes for instance, they cannot bring as many raised-lined maps as they want because it takes times to prepare and because they are cumbersome. However, they could bring as many as they want with a tablet combining VibHand.

Mathematical graphs. Mathematical graphs, and specifically line graphs, are very often used in classes. With VibHand, users could easily explore a digital version of a line graph and carry usual data analytics tasks, such as looking for max and min values, or compare two-line graphs (similar to the comparison task in our study).

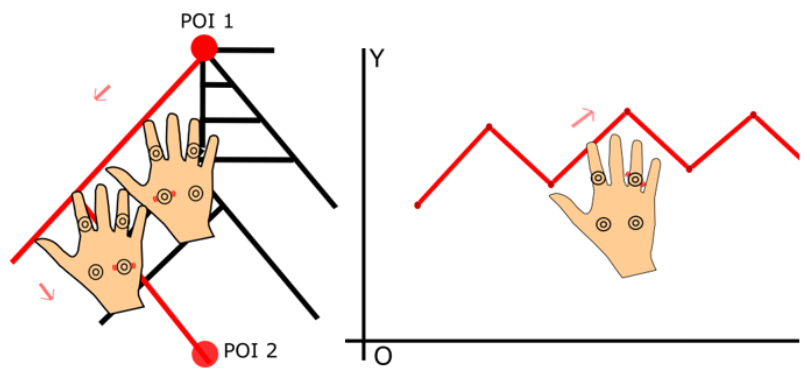

Fig. 14. A user exploring a path between two POls on a neighborhood map (left) and a line plot on a mathematical graph (right) with VibHand.

4.9.4 Limitations and Future work. Overall, these results show that VibHand improves the efficiency of tactile exploration as well as the mental representation of the explored graphics. An important question is to compare the exploration with VibHand to the exploration with regular raised-line graphics. This question should be addressed in future work. We do not believe that VibHand would outperform raised-line graphics, but we make the hypothesis that VibHand would provide VI users with ubiquitous useful and convenient access to digital graphics in many real-life contexts. Such a comparison should then not only address the compared usability of the two techniques but also the ubiquity of the techniques with different graphics (maps, mathematical graphs, etc.) and different tasks to be achieved (exploration, recognition, comparison, decision making, etc.).

Another important point to be addressed in a future work is the integration of VibHand in a more ergonomic device. The current VibHand prototype is made of four vibrators connected to an Arduino, which is connected to the tablet with network. The next prototype could be a mitt that contains the vibrators and microcontrollers.

In addition, the current design of VibHand includes eight cardinal directions only. Therefore, it can only work with simplified graphics (similar to the most common raised-line graphics used in special education center). However, providing more directions could give access to more 
complex graphics, but at the same time increase the cognitive load associated to the exploration and the cognitive integration of the graphic. In the future, we plan to investigate the possibility of extending the number of vibrotactile cues to provide more directions. We plan to investigate the use of tactile illusions [20], such as the phantom sensation [2]. Previous work showed that it is possible to generate a virtual vibration between two real vibrations [27]. This could provide VibHand with more directions without adding vibrators, but further studies are needed to evaluate if these vibrotactile illusions can be used for exploration and do not raise any cognitive issues.

\section{CONCLUSION}

In this paper, we presented VibHand, a promising non-visual vibrotactile interface for providing a better access to digital graphics on tablets. VibHand extends regular tablet vibrations by providing both directional and progression cues using four vibrators on the back of the hand. Our design is based on a set of formative iterations leading to a summative study with 12 blindfolded participants and 12 participants with VI. The results confirm the advantages of VibHand on digital graphic exploration speed, recognition accuracy and user preference. Our comparison of BF and VI groups revealed no difference on the identification accuracy of known graphics, nor on the comparison accuracy. However, VI participants take less time and have shorter unrelated finger paths than BF participants.

\section{ACKNOWLEDGMENTS}

We thank all the participants, the laboratory "Cherchons pour Voir" and China Scholarship Council.

\section{REFERENCES}

[1] Jérémy Albouys-Perrois, Jérémy Laviole, Carine Briant, and Anke M. Brock. 2018. Towards a Multisensory Augmented Reality Map for Blind and Low Vision People: a Participatory Design Approach. In Proceedings of the 2018 CHI Conference on Human Factors in Computing Systems (CHI '18). ACM, New York, NY, USA, Paper 629, 14 pages. DOI: https://doi.org/10.1145/3173574.3174203

[2] David S. Alles. 1970. Information Transmission by Phantom Sensations. IEEE Trans. Man-Machine Syst. 11, 1 (1970), 85-91. DOI:https://doi.org/10.1109/TMMS.1970.299967

[3] Jessalyn Alvina, Shengdong Zhao, Simon T. Perrault, Maryam Azh, Thijs Roumen, and Morten Fjeld. 2015. OmniVib: Towards Cross-body Spatiotemporal Vibrotactile Notifications for Mobile Phones. In Proceedings of the 33rd Annual ACM Conference on Human Factors in Computing Systems (CHI '15). ACM, New York, NY, USA, 2487-2496. DOI: https://doi.org/10.1145/2702123.2702341

[4] Heller, M.A., 1989. Picture and pattern perception in the sighted and the blind: the advantage of the late blind. Perception, 18(3), pp.379-389

[5] Sandra Bardot, Marcos Serrano, and Christophe Jouffrais. 2016. From tactile to virtual: using a smartwatch to improve spatial map exploration for visually impaired users. In Proceedings of the 18th International Conference on Human-Computer Interaction with Mobile Devices and Services (MobileHCI '16). ACM, New York, NY, USA, 100-111. DOI: https://doi.org/10.1145/2935334.2935342

[6] Anke Brock, Samuel Lebaz, Bernard Oriola, Delphine Picard, Christophe Jouffrais, and Philippe Truillet. 2012. Kin'touch: understanding how visually impaired people explore tactile maps. In CHI '12 Extended Abstracts on Human Factors in Computing Systems (CHI EA '12). ACM, New York, NY, USA, 2471-2476. DOI: https://doi.org/10.1145/2212776.2223821

[7] Emeline Brulé, Brianna J. Tomlinson, Oussama Metatla, Christophe Jouffrais, and Marcos Serrano. 2020. Review of Quantitative Empirical Evaluations of Technology for People with Visual Impairments. In Proceedings of the 2020 CHI Conference on Human Factors in Computing Systems (CHI '20). Association for Computing Machinery, New York, NY, USA, 1-14. DOI:https://doi.org/10.1145/3313831.3376749

[8] Jessica R. Cauchard, Janette L. Cheng, Thomas Pietrzak, and James A. Landay. 2016. ActiVibe: Design and Evaluation of Vibrations for Progress Monitoring. In Proceedings of the 2016 CHI Conference on Human Factors in Computing Systems (CHI '16). Association for Computing Machinery, New York, NY, USA, 3261-3271. DOI:https://doi.org/10.1145/2858036.2858046

[9] Cornoldi Cesare, Carla Tinti, Irene C. Mammarella, Anna Maria Re, and Diego Varotto. "Memory for an imagined pathway and strategy effects in sighted and in totally congenitally blind individuals." Acta Psychologica 130, no. 1 (2009): 11-16. 
[10] Angela Chang, Sile O'Modhrain, Rob Jacob, Eric Gunther, and Hiroshi Ishii. 2002. ComTouch: design of a vibrotactile communication device. In Proceedings of the 4th conference on Designing interactive systems: processes, practices, methods, and techniques (DIS '02). ACM, New York, NY, USA, 312-320. DOI=http://dx.doi.org/10.1145/778712.778755

[11] Qin Chen, Simon T. Perrault, Quentin Roy, and Lonce Wyse. 2018. Effect of temporality, physical activity and cognitive load on spatiotemporal vibrotactile pattern recognition. In Proceedings of the 2018 International Conference on Advanced Visual Interfaces (AVI '18). ACM, New York, NY, USA, Article 25, 9 pages. DOI: https://doi.org/10.1145/3206505.3206511

[12] Camors Damien, Damien Appert, Jean-Baptiste Durand, and Christophe Jouffrais. "Tactile Cues for Improving Target Localization in Subjects with Tunnel Vision." Multimodal Technologies and Interaction 3, no. 2 (2019): 26.

[13] Jacobson, R. Dan. "Navigating maps with little or no sight: An audio-tactile approach." In Content Visualization and Intermedia Representations (CVIR'98). 1998.

[14] Delphine Picard, Samuel Lebaz, Christophe Jouffrais, and Catherine Monnier. "Haptic recognition of twodimensional raised-line patterns by early-blind, late-blind, and blindfolded sighted adults." Perception 39, no. 2 (2010): 224-235

[15] Julie Ducasse, Anke M Brock, and Christophe Jouffrais. 2018. Accessible Interactive Maps for Visually Impaired Users. In Mobility of Visually Impaired People. Springer, 537-584. DOI:https://doi.org/10.1007/978-3-319-54446$5 \quad 17$

[16] Julie Ducasse, Marc J-M Macé, Marcos Serrano, and Christophe Jouffrais. 2016. Tangible Reels: Construction and Exploration of Tangible Maps by Visually Impaired Users. In Proceedings of the $2016 \mathrm{CHI}$ Conference on Human Factors in Computing Systems (CHI '16). ACM, New York, NY, USA, 2186-2197. DOI: https://doi.org/10.1145/2858036.2858058

[17] Don Samitha Elvitigala, Denys J. C. Matthies, Vipula Dissanayaka, Chamod Weerasinghe, and Suranga Nanayakkara. 2019. 2bit-TactileHand: Evaluating Tactons for On-Body Vibrotactile Displays on the Hand and Wrist. In Proceedings of the 10th Augmented Human International Conference 2019 (AH2019). ACM, New York, NY, USA, Article 3, 8 pages. DOI: https://doi.org/10.1145/3311823.3311832

[18] Nicholas A. Giudice, Hari Prasath Palani, Eric Brenner, and Kevin M. Kramer. 2012. Learning non-visual graphical information using a touch-based vibro-audio interface. In Proceedings of the 14th international ACM SIGACCESS conference on Computers and accessibility (ASSETS '12). ACM, New York, NY, USA, 103-110. DOI: https://doi.org/10.1145/2384916.2384935

[19] Cagatay Goncu and Kim Marriott. 2011. GraVVITAS: Generic Multi-touch Presentation of Accessible Graphics. In Proceedings of the 13th IFIP TC 13 Conference on Human-Computer Interaction (INTERACT '11),30-48. DOI:https://doi.org/10.1007/978-3-642-23774-4

[20] Jonggi Hong, Alisha Pradhan, Jon E. Froehlich, and Leah Findlater. 2017. Evaluating Wrist-Based Haptic Feedback for Non-Visual Target Finding and Path Tracing on a 2D Surface. In Proceedings of the 19th International ACM SIGACCESS Conference on Computers and Accessibility (ASSETS '17). Association for Computing Machinery, New York, NY, USA, 210-219. DOI:https://doi.org/10.1145/3132525.3132538

[21] Sreekar Krishna, Shantanu Bala, Troy McDaniel, Stephen McGuire, and Sethuraman Panchanathan. 2010. VibroGlove: an assistive technology aid for conveying facial expressions. In CHI '10 Extended Abstracts on Human Factors in Computing Systems (CHI EA '10). ACM, New York, NY, USA, 3637-3642. DOI: https://doi.org/10.1145/1753846.1754031

[22] Zhuoluo Ma, Yue Liu, Dejiang Ye, and Lu Zhao. 2019. Vibrotactile Wristband for Warning and Guiding in Automated Vehicles. In Extended Abstracts of the 2019 CHI Conference on Human Factors in Computing Systems (CHI EA '19). ACM, New York, NY, USA, Paper LBW2220, 6 pages. DOI https://doi.org/10.1145/3290607.3312819

[23] Jonatan Martínez, Arturo García, Miguel Oliver, José Pascual Molina, and Pascual González. 2016. Identifying Virtual 3D Geometric Shapes with a Vibrotactile Glove. IEEE Comput. Graph. Appl. 36, 1 (2016), 42-51. DOI:https://doi.org/10.1109/MCG.2014.81

[24] Anita Meier, Denys J. C. Matthies, Bodo Urban, and Reto Wettach. 2015. Exploring vibrotactile feedback on the body and foot for the purpose of pedestrian navigation. In Proceedings of the 2nd International Workshop on Sensor-based Activity Recognition and Interaction, ACM, 1-11. DOI:https://doi.org/10.1145/2790044.2790051

[25] Giuseppe Melfi, Karin Müller, Thorsten Schwarz, Gerhard Jaworek, and Rainer Stiefelhagen. 2020. Understanding what you feel: A Mobile Audio-Tactile System for Graphics Used at Schools with Students with Visual Impairment. In Proceedings of the 2020 CHI Conference on Human Factors in Computing Systems (CHI '20). Association for Computing Machinery, New York, NY, USA, 1-12. DOI:https://doi.org/10.1145/3313831.3376508

[26] Hari P. Palani, Paul D. S. Fink \& Nicholas A. Giudice (2020): Design Guidelines for Schematizing and Rendering Haptically Perceivable Graphical Elements on Touchscreen Devices, International Journal of Human-Computer Interaction, 1-22.

[27] Jaeyoung Park, Jaeha Kim, Yonghwan Oh, and Hong Z Tan. 2016. Rendering moving tactile stroke on the palm using a sparse 2d array. In International Conference on Human Haptic Sensing and Touch Enabled Computer Applications. Springer, Cham, 47-56. DOI:https://doi.org/10.1007/978-3-319-42324-1

[28] Benjamin Poppinga, Charlotte Magnusson, Martin Pielot, and Kirsten Rassmus-Gröhn. 2011. TouchOver map audio-tactile exploration of interactive maps. In Proceedings of the 13th International Conference on Human 
Computer Interaction with Mobile Devices and Services (MobileHCI '11). ACM, New York, NY, USA, 545-550. DOI: https://doi.org/10.1145/2037373.2037458

[29] R.M. Sakia. The Box-Cox transformation technique: a review. Journal of the Royal Statistical Society: Series D (The Statistician), 41(2), 169-178.

[30] Elizabeth B-N Sanders. 2002. From user-centered to participatory design approaches. Design and the social sciences: Making connections 1, 8 (2002).

[31] Choi Seungmoon, and Katherine J. Kuchenbecker. "Vibrotactile display: Perception, technology, and applications." Proceedings of the IEEE 101, no. 9 (2012): 2093-2104.

[32] Abigale Stangl, Meredith Ringel Morris, and Danna Gurari. 2020. "Person, Shoes, Tree. Is the Person Naked?" What People with Vision Impairments Want in Image Descriptions. In Proceedings of the 2020 CHI Conference on Human Factors in Computing Systems (CHI '20). Association for Computing Machinery, New York, NY, USA, 113. DOI:https://doi.org/10.1145/3313831.3376404

[33] Anil K. Raj, Steven J. Kass, and James F. Perry. 2012. Vibrotactile Displays for Improving Spatial Awareness. In Proceedings of the Human Factors and Ergonomics Society Annual Meeting. Sage CA: Los Angeles, 44, 1 (2012), 181-184. DOI:https://doi.org/10.1177/154193120004400148

[34] Joe Tekli, Youssef Bou Issa, and Richard Chbeir. 2018. Evaluating touch-screen vibration modality for blind users to access simple shapes and graphics. International Journal of Human-Computer Studies 110, August 2017 (2018), 115-133. DOI:https://doi.org/10.1016/j.ijhcs.2017.10.009

[35] Zheshen Wang, Baoxin Li, Terri Hedgpeth, and Teresa Haven. 2009. Instant tactile-audio map: enabling access to digital maps for people with visual impairment. In Proceedings of the 11th international ACM SIGACCESS conference on Computers and accessibility (Assets '09). ACM, New York, NY, USA, 43-50. DOI: https://doi.org/10.1145/1639642.1639652

[36] Ruolin Wang, Chun Yu, Xing-Dong Yang, Weijie He, and Yuanchun Shi. 2019. EarTouch: Facilitating Smartphone Use for Visually Impaired People in Mobile and Public Scenarios. In Proceedings of the 2019 CHI Conference on Human Factors in Computing Systems (CHI '19). ACM, New York, NY, USA, Paper 24, 13 pages. DOI: https://doi.org/10.1145/3290605.3300254

[37] John S Zelek. 2005. Seeing by touch (haptics) for wayfinding. In International congress series, Vol. 1282. Elsevier, 1108-1112. 